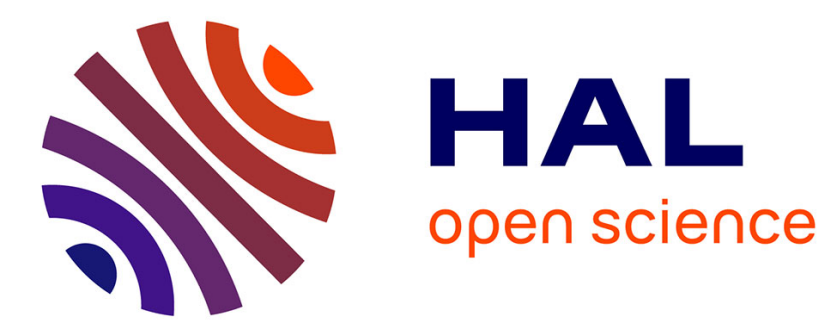

\title{
Dynamics of genotypes in a farm recovered from an outbreak of Glässer's Disease
}

A. Olvera, M. Cerdà-Cuéllar, M. Nofrarías, E. Revilla, J. Segalés, V. Aragon

\section{To cite this version:}

A. Olvera, M. Cerdà-Cuéllar, M. Nofrarías, E. Revilla, J. Segalés, et al.. Dynamics of genotypes in a farm recovered from an outbreak of Glässer's Disease. Veterinary Microbiology, 2007, 123 (1-3), pp.230. 10.1016/j.vetmic.2007.03.004 . hal-00532221

\section{HAL Id: hal-00532221 \\ https://hal.science/hal-00532221}

Submitted on 4 Nov 2010

HAL is a multi-disciplinary open access archive for the deposit and dissemination of scientific research documents, whether they are published or not. The documents may come from teaching and research institutions in France or abroad, or from public or private research centers.
L'archive ouverte pluridisciplinaire HAL, est destinée au dépôt et à la diffusion de documents scientifiques de niveau recherche, publiés ou non, émanant des établissements d'enseignement et de recherche français ou étrangers, des laboratoires publics ou privés. 


\section{Accepted Manuscript}

Title: Dynamics of Haemophilus parasuis genotypes in a farm recovered from an outbreak of Glässer's Disease

Authors: A. Olvera, M. Cerdà-Cuéllar, M. Nofrarías, E. Revilla, J. Segalés, V. Aragon

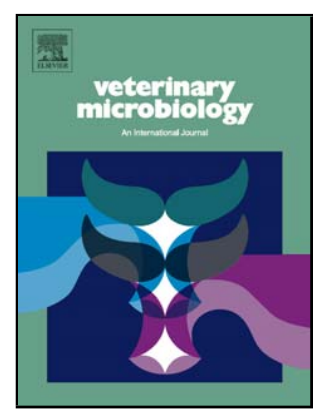

PII: $\quad$ S0378-1135(07)00137-X

DOI: doi:10.1016/j.vetmic.2007.03.004

Reference: $\quad$ VETMIC 3614

To appear in: $\quad$ VETMIC

Received date: $\quad$ 7-2-2007

Revised date: $\quad 1-3-2007$

Accepted date: $\quad$ 8-3-2007

Please cite this article as: Olvera, A., Cerdà-Cuéllar, M., Nofrarías, M., Revilla, E., Segalés, J., Aragon, V., Dynamics of Haemophilus parasuis genotypes in a farm recovered from an outbreak of Glässer's Disease, Veterinary Microbiology (2007), doi:10.1016/j.vetmic.2007.03.004

This is a PDF file of an unedited manuscript that has been accepted for publication. As a service to our customers we are providing this early version of the manuscript. The manuscript will undergo copyediting, typesetting, and review of the resulting proof before it is published in its final form. Please note that during the production process errors may be discovered which could affect the content, and all legal disclaimers that apply to the journal pertain. 
4 Dynamics of Haemophilus parasuis genotypes in a farm recovered from an 5 outbreak of Glässer's Disease

8 Running Title: Dynamics of $H$. parasu is strains genotypes

10 A. Olvera ${ }^{1}$, M. Cerdà-Cuéllar ${ }^{1}$, M. Nofrarías ${ }^{1}$, E. Revilla ${ }^{2}, J_{\text {. Segalés }}{ }^{1,3}$ and V. Aragon ${ }^{{ }^{*}}$

$12{ }^{1}$ Centre de Recerca en Sanitat Animal. Edifici CReSA. Campus de Bellaterra-UAB. 13 08193-Bellaterra. Barcelona. Spain.

$14 \quad{ }^{2}$ Albet, S.A. Perot Rocaguinarda 18. 08500-Vic. Barcelona. Spain

$15{ }^{3}$ Department Sanitat i Anatomia Animals. Universitat Autònoma de Barcelona. Campus 16 de Bellaterra. 08193-Bella terra. Barcelona. Spain.

18 * Corresponding author: Virginia Aragon

19 Centre de Recerca en Sanitat Animal (CReSA). Campus de Bellaterra-Universitat 20 Autònoma de Barcelona. 08193-Bellaterra, Barcelona (Spain).

21 Phone: +34935814494

22 Fax: +34935814490

23 e-mail: virginia.aragon@cresa.uab.es 


\section{Abstract}

25 Haemophilus parasuis is a colonizer of the upper respiratory tract of pigs, although it is

26 better known as the etiological agent of Glässer's disease. Interestingly, several strains

27 can be isolated from a single farm, as determined by both genotyping and serotyping.

28 However, it is not known how an outbreak and the subsequent treatment affect the 29 population of $H$. parasuis strains. In this study, a farm was studied during an outbreak 30 of Glässer's disease and one year after antimicrobial treatment and elimination of 31 clinical signs. Bacterial isolation was attempted from nasal swabs and lesions. After 32 isolation, antimicrobial susceptibility, serotype and genotype were determined. Two 33 different genotyping techniques, enterobacterial repetitive intergenic consensus (ERIC)34 PCR and multilocus sequence typing (MLST) were used. The H. parasuis strain that was isolated from lesions during the disease outbreak clustered with other virulent strains by both MLST and serotyping analysis. Nasal isolates were included in the

37 correspond ing nasal cluster by MLST, but they presen ted high variability by serotyping.

38 These nasal isolates included serotypes previously classified as virulent and non-

39 virulent. Finally, we found that during the antimicrobial treatment the diversity of

40 strains isolated in the farm was affected and just one strain, which was resistant to the

41 treatment, was detected. One year after the treatment strain diversity was back to normal 42 (3 strains).

44 Keywords: Haemophilus parasuis, Glässer's disease, field strains, genotyping 


\section{Introduction}

Haemophilus parasuis is the etiological agent of Glässer's disease in pigs, which is characterized by serofibrinous to fibrinopurulent polyserositis, arthritis and meningitis (Nicolet, 1968). H. parasuis is also involved in other clinico-pathological outcomes, such as pneumonia and sudden death, and causes high morbidity and mortality in naïve swine populations (Miniats et al., 1986). On the other hand, H. parasuis colonizes de upper respiratory tract of pigs at a very young age and, therefore, it can be isolated from the nose of healthy pigs (Harris et al., 1969; Møller and Kilian, 1990; Oliveira and Pijoan, 2004).

H. parasuis strains differ in several features, including pathogenic potential (RappGabrielson et al., 2006). Classically, strains of $H$. parasuis have been classified by serotyping and, although some serotypes seem to be more pathogenic than others (Rosner et al., 1991; Rapp-Gabrielson et al., 1992; Nielsen, 1993; Amano et al., 1994, Amano et al., 1996), the correlation between serotype and virulence is not complete. More importantly, a significant percentage of isolates, between 15 and 41\%, are nontypeable by serotyping (Oliveira and Pijoan, 2004). In order to circumvent this problem, fingerprinting and sequencing methods have been developed to assess the heterogeneity of $H$. parasuis isolates by genotyping (Olvera et al., 2006c). The application of enterobacterial repetitive intergenic consensus (ERIC)-PCR to $H$. parasuis is especially useful in local epidemiology (i.e. to assess the strains circulating within a farm) (Ruiz et al., 2001; Oliveira et al., 2003), but the results of this technique are hardly portable and difficult to compare among laboratories. Thus, in order to perform global epidemiology studies, other genotyping methods have been developed (Olvera et al., 2006a; Olvera et al., 2006b). Sequence typing, especially multilocus sequence typing (MLST), reported 
69

clusters of $H$. parasuis strains associated with disease production or, on the contrary, with nasal microbiota (Olvera et al., 2006b).

Using different genotyping methods, it has been shown that several strains can be isolated from a single conventional farm and that strains associated with respiratory pathology differ from systemic ones (Smart et al., 1988; Smart et al., 1993; Ruiz et al., 2001; Oliveira et al., 2003; Olvera et al., 2006a; Olvera et al., 2006b). However, it is not known how an episode of Glässer's disease and the subsequent treatment with antimicrobials affect the population of $H$. parasuis strains in a farm. Thus, the goal of this work was to study $H$. parasuis strain dynamics in a farm during an outbreak of Glässer's disease and a year later, using serotyping and two different genotyping techniques.

\section{Material and Methods}

\section{Case farm}

In October 2004, an outbreak of Glässer's dis ease was diagnosed in a 300-sow, farrowto-finish farm located in Northeastern Spain. Clinical signs, including dyspnoea, diarrhoea and sudden death, were observed at about 5 weeks of age (weaning was performed at $\sim 24$ days of age). On-farm necropsies showed fibrinous polyserositis, and lungs from four affected pigs were submitted for bacterial isolation to the diagnostic service of the Department of Infectious Diseases of the Veterinary School at the Universitat Autònoma de Barcelona (UAB) in Spain. H. parasuis was isolated from one of these lungs (isolate 228/04), and antimicrobial sensitivity determination was performed. The veterinarian decided to start a treatment with amoxicillin (feed treatment, $400 \mathrm{ppm})$. 
94 After one month of amoxicillin treatment (November 2004), a similar clinical95 pathological problem (with fibrinous polyserositis) was still present in the farm, but 96 affecting the growing units (9-11 week-old pigs). Therefore, the antimicrobial treatment

97 was changed and a combination of phenoximetilpenicilin and tylosin was applied (feed treatment, $200 \mathrm{ppm}$ and $100 \mathrm{ppm}$, respectively). At that time, two 9 week-old pigs

99 (CD12 and CD13) were found dead and, at necropsy, sero-fibrinous fluids of thoracic

100 and abdominal cavities were taken for bacterial isolation. Besides, eleven nasal swabs

101 (CD1 to CD11), sampling both nostrils, were taken from 6 to 11 week-old pigs from the 102 units with clinical disease. The established antimicrobial treatment apparently worked 103 and no further cases compatible with Glässer's disease were reported.

104 One year after the initial outbreak (October 2005), the farm remained free of Glässer's 105 disease based on clinical and pathological observations. At that time, ten nasal swabs 106 from 6 to 8 week-old pigs (CN1 to $\mathrm{CN} 10)$ were taken for $H$. parasuis isolation and the 107 subsequent typing analysis.

\section{H. parasuis is olation and identification}

110 Collected swabs were placed in Amies medium and transported under refrigeration to

111 the laboratory, where they were plated on chocolate agar to isolate colonies. After 2 to 3

112 days at $37^{\circ} \mathrm{C}$ with $5 \% \mathrm{CO}_{2}$, all $\mathrm{H}$. parasuis-like colonies were selected and subcultured

113 for further analysis. A species-specific PCR (Oliveira et al., 2001) and classical

114 biochemical tests (Møller and Kilian, 1990) to identify H. parasuis were performed,

115 although final identification was accomplished by $16 \mathrm{~S}$ rRNA gene sequencing

116 (GenBank accession numbers DQ228977, DQ228980, DQ228981, DQ228983 to

117 DQ228985, EF396294, EF396297 to EF396303). Bacterial suspensions in sterile

118 phosphate buffer saline (PBS) were used to extract genomic DNA from each isolate. 
119 The extraction was performed with the Instagene DNA purification Matrix 2 (Bio-Rad

120 Laboratories Inc., Spain) following manufacturer instructions. A fragment of the $16 \mathrm{~S}$

121 rRNA gene of about $1400 \mathrm{bp}$ was amplified and sequenced as reported previously

122 (Olvera et al., 2006a). The resulting sequences were used in database searches using the

123 BLAST algorithm (Altschul et al., 1997). Both the NCBI

124 (http://www.ncbi.nlm.nih.gov/BLAST) and the Ribosomal Database Project

125 (http://rdp.cme.msu.edu) databases were searched. A threshold of 99\% sequence

126 identity was used for species identification (Janda and Abbott, 2002).

128 Antimicrobial susceptibility

129 Antimicrobial susceptibility tests were performed using the Kirby-Bauer method

130 according to CLSI guideline M31-A2, with some modifications. Briefly, a 0.5

131 MacFarland suspension of each isolate was made from a $24 \mathrm{~h}$ culture on chocolate agar

132 plates. Each suspension was spread on chocolate agar plates using a swab and discs of

133 seven different antimicrobials (Neo-sensitabs ${ }^{\mathrm{TM}}$, Rosco Diagnostica, Denmark) were

134 placed on the plates, which were then incubated for $24 \mathrm{~h}$ at $37^{\circ} \mathrm{C}$ with $5 \% \mathrm{CO}_{2}$. The

135 antimicrobials tested were amoxicillin $(30 \mu \mathrm{g})$, ceftifour $(30 \mu \mathrm{g})$, enrofloxacin $(10 \mu \mathrm{g})$,

136 doxycycline $(80 \mu \mathrm{g})$, trimethoprim-sulphomethoxazole $(5.2+240 \mu \mathrm{g})$ and tylosin $(150$

$137 \mu \mathrm{g})$.

138

139 Serotyping

140 Serotype determination was performed by indirect hemagglutination at the Department

141 of Sanidad Animal of the Veterinary School at the University of Leon (Spain) following

142 a previously published protocol (del Rio et al., 2003). Soluble antigen was obtained 
143 after boiling a bacterial suspension and subsequent centrifugation to eliminate insoluble

144 debris.

145

146 Multilocus sequence typing (MLST)

147 Fragments of the $r p o B, 6 p g d, m d h, i n f B, f r d B, g 3 p d$ and atpD genes of $H$. parasuis were

148 amplified as previously published (Olvera et al., 2006a). Amplicons were sequenced

149 using the same PCR primers and BigDye terminator v.3.1 kit, using an ABI 3100 DNA

150 sequencer (Applied Biosystems, USA). Sequences were deposited at the GenBank

151 database (accession numbers DQ781634 to DQ781637, DQ781660, DQ781661,

152 DQ781663, DQ781666, DQ781673, DQ781503 to DQ781506, DQ781529, DQ781530,

153 DQ781532, DQ781535, DQ781542, DQ782289 to DQ782292, DQ782315, DQ782316,

154 DQ782318, DQ782321, DQ782328, DQ782158, DQ782159, DQ782184, DQ782185,

155 DQ782187, DQ782190, DQ782197, DQ782027 to DQ782030, DQ782053, DQ782054,

156 DQ782056, DQ782059, DQ782066, DQ782896 to DQ782899, DQ78292, DQ78293,

157 DQ782925, DQ782928, DQ782935, DQ782765 to DQ782768, DQ782791, DQ782792,

158 DQ782794, DQ782797, DQ782804, EF396288 to EF396293, EF396308 to EF396322).

159 Sequence editing and allele assignment was performed with Fingerprinting II v3.0

160 software (BioRad, USA). MLST analysis was carried out using START2 (Jolley et al.,

1612001 ) by the neighbour-joining algorithm (NJ) based on allelic profiles.

162

163 Enterobacterial repetitive intergenic consensus (ERIC)-PCR

164 Purified DNA was quantified by spectrometry and $100 \mathrm{ng}$ were used as template in

165 ERIC-PCR. The technique followed a previously published protocol (Oliveira et al.,

166 2003), including an extra final extension step of $20 \mathrm{~min}$ (Olvera et al., 2006a). Images

167 of the gels were captured with a ChemiGenius transiluminator (SynGene Ltd. Frederick, 
MD, USA.) and stored as TIFF files for further analysis. Bands from 4,000 to $100 \mathrm{bp}$

169 were used in the analysis. ERIC-PCR fingerprints analysis, sequence editing and

170 analysis were performed using the Fingerprinting II v3.0 software. Finally, ERIC-PCR

171 band patterns were normalized and a Pearson correlation similarity matrix calculated.

172 Cluster analysis of ERIC-PCR fingerprints were performed by UPGMA (Ooyen, 2001).

\section{Results and discussion}

176 Several studies have examined the variability of $H$. parasuis strains circulating in a farm

177 by genotyping and serotyping, but none of them has followed up the strain population

178 over time. Moreover, there are several studies on the antimicrobial susceptibility of $H$.

179 parasuis, but, as far as we know, this is the only work that studies the effect of

180 antimicrobial treatment on $H$. parasuis strain variability within the same farm.

181 When disease outbreak was observed (October 2004), H. parasuis was isolated from

182 one of the 4 lungs from sacrificed animals with fibrinous polyserositis (isolate 228/04).

183 Antimicrobial susceptibility tests showed that isolate $228 / 04$ was sensitive to all the

184 antimicrobials tested (Table 1). Amoxicillin treatment was implemented but, after one

185 month (November 2004), the clinical problem moved to the growing units. Two animals

186 (CD12 and CD13) were found dead and after necropsia, H. parasuis DNA was detected

187 by PCR in abdominal and thoracic fluids. However, H. parasuis could not be isolated

188 from the lesions of these pigs. These results can be explained by the fastidious growth

189 requirements of this microorganism and its difficult isolation from already dead

190 animals. At that time, a total of $25 \mathrm{H}$. parasuis isolates were obtained from eleven nasal

191 swabs (isolates CD1 to CD11). Genotyping of these nasal isolates showed that they had

192 the same ERIC-PCR profile (Fig. 1), no differences in 16S rRNA gene sequence (data 
not shown) and the same MLST sequence type (ST) (Fig. 1), indicating that they were a

194 single strain. The antimicrobial susceptibility tests of several $H$. parasuis CD isolates

195 demonstrated that those isolates were highly resistant to amoxicillin and sensitive to the

196 rest of antimicrobials tested (Table 1). Antimicrobial treatment had a clear effect on the

197 population of $H$. parasuis in this farm. Clearly, the use of amoxicillin to control the

198 outbreak selected one clone (H. parasuis $\mathrm{CD})$ that was resistant to this antimicrobial

199 and induced a homogenization of the strain population. Apparently, strain 228/04 was

200 eliminated by the treatment, since it was not isolated anymore.

201 Besides antimicrobial susceptibility, H. parasuis CD isolates and strain 228/04 showed

202 clear genotypic differences (Fig. 1), indicating that the $H$. parasuis CD strain and

$203228 / 04$ were indeed different strains. These results may ind icate that two strains, 228/04

204 and $H$. parasuis $\mathrm{CD}$, were initially associated with clinical problems and the

205 antimicrobial treatment eliminated just one or, alternatively, that the elimination of the

206 first one (228/04) was an opportunity for the second one (CD) to proliferate and cause a

207 second round of disease. On the other hand, it can not be ruled out that the first one was

208 never the cause of the disease. Actually, 228/04 was isolated from lung and a clear link

209 with the observed systemic disease is not evident, since a lung isolate may not

210 necessarily be the cause of a septicaemia. This is a major problem with all $H$. parasuis

211 lung isolates from Glässer's disease cases, together with the difficulties to discard them

212 as postmortem invasion from the upper respiratory tract. The global comparison of

213 strains by MLST (Fig 2) supported that 228/04 was indeed the cause of the disease since

214 it was included in a cluster associated with disease production and formed mainly by

215 systemic isolates (Olvera et al., 2006b). Moreover, this strain belonged to serotype 5,

216 which has been repeatedly demonstrated highly virulent in experimental infections

217 (Kielstein and Rapp-Gabrielson, 1992; Nielsen, 1993; Amano et al., 1994). On the other 
218 hand, H. parasuis CD isolates were included in a MLST cluster with strains of nasal

219 origin (Fig. 2), indicating that they were probably a non-virulent strain not implicated in

220 the clinical outbreak. Curiously, when the serotype was determined for 3 of those

221 isolates, they belonged to serotypes 10,14 and 15; although they had the same genotype

222 (Fig 1). Taking in to account the rate of evolution of ERIC-PCR and MLST patterns this

223 could mean that serotype in $H$. parasuis is an unstable phenotypic characteristic that can

224 change relatively fast, at least faster than genomic rearrangements or mutations.

225 Alternatively, H. parasuis serotyping needs further optimization to avoid this

226 variability, which could be due to a certain degree of crossreactivity between serotypes.

227 Although no other H. parasuis strain was isolated in the affected growing units during

228 the clinical problem, association of $H$. parasuis $\mathrm{CD}$ strain with disease onset is,

229 obviously, difficult to establish because it was isolated from nasal swabs. Unfortunately,

230 no $H$. parasuis could be isolated from the lesions of pigs CD12 and CD13, although the

231 bacterium was detected by the $H$. parasuis-specific PCR. Unexpectedly, ascites from

232 pig CD12 yielded a Moraxella spp isolate (CD12CA4), as identified by 16S rRNA

233 gene sequencing (best Blastn hit with Moraxella cuniculi [Accession number

234 AF005188] with $97 \%$ of sequence identity). This result indicates the possibility that the

235 clinical signs observed in the growing units were due to this opportunistic pathogen.

236 Nevertheless, the association of Moraxella spp with disease onset in pigs is difficult to

237 establish. Some authors have reported the isolation of Moraxella-like microorganisms

238 from polyserositis lesions of pigs, but the pathogenicity of these isolates could not be

239 demonstrated in experimental infections (Larsen et al., 1973; Nielsen and Danielsen,

240 1975). 
241 Finally, the treatment was changed to phenoximethylpenicillin and tylosin and the 242 problem was controlled (all the isolates of this study were sensitive, at least, to tylosin) 243 and the $H$. parasuis CD strain was eliminated.

244 After one year, in which the farm remained free of Glässer's disease, ten 6 to 8-week245 old pigs were sampled and a total of 23 isolates of $H$. parasuis were obtained (isolates $246 \mathrm{CN}$ followed by numbers). Three different strains were identified among these $\mathrm{CN}$ 247 isolates (represented by CN9-2, CN10-1 and the group of CN8-1; Fig 1) by both 248 genotyping methods (ERIC-PCR and MLST). Thus, the variability of strains was back 249 to normal (usually, 3-5 strains/farm) (Smart et al., 1988; Smart et al., 1989; Smart et al., 250 1993; Rafiee et al., 2000). When the MLST analysis was done with 205 strains from 251 different origins, all $\mathrm{CN}$ isolates were included in the cluster of strains associated with 252 nasal isolation (Fig. 2) (Olvera et al., 2006b). Serotype was determined for a 253 representative of each genotype and serotypes 9 and 15 were reported (Fig. 1). These 254 isolates showed different susceptibilities to amoxicillin (Table 1), but all of them were 255 sensitive to the rest of antimicrobials tested.

256 CN10-1 was resistant to amoxicillin (Table 1) and showed the closest genotype to the 257 CD isolates, with just one different allele (infB 16) in the MLST allelic profile. Allele 258 infB 16 was also present in the isolates from the group of CN8-1. The CN10-1 infB 259 allele (infB 16) differed in two nucleotides with the corresponding allele in the CD 260 strains (inf 8) and was identical to the CN8-1 infB allele. The detection of homologous 261 recombination in $H$. parasuis in a previous work (Olvera et al., 2006b) gives more 262 support to the occurrence of one homologous recombination event between the CD and 263 the CN8-1 isolates, instead of two independent point mutation events. Indeed, it is very 264 likely that CN10-1 is a descendent of the H. parasuis CD strain that has exchanged the 265 infB 16 allele with the CN8-1 isolates. The origin of the remaining $\mathrm{CN}$ strains 
266 (represented by isolates CN2-1 and CN9-2) is not fully clear given that they were very

267 different from those detected during the clinical outbreak. It is possible that those $\mathrm{CN}$

268 strains were in the farm prior to the outbreak. Thus, the use of antimicrobial treatment

269 induced the elimination of them in the units were the treatment was applied, but they

270 were reintroduced by the sows. Therefore, they might represent the situation of the farm

271 before the clinical outbreak. However, it cannot be ruled out that those $\mathrm{CN}$ strains were

272 introduced by replacement breed ing stock.

273 The genotyping methods used in this study, ERIC-PCR and MLST, were useful in the

274 assessment of the number of clones present in a farm, and yielded similar results. For

275 the identification of the different clones in a farm, ERIC-PCR is faster and less

276 expensive than MLST. However, MLST produces additional information, since it also

277 allows the comparison of the iso lates in a particular farm with other epidemiologically-

278 unrelated isolates. The comparison of the isolates obtained in the case farm with strains

279 from different clinical and geographical origins (Olvera et al., 2006b) was helpful to

280 elucidate the putative virulence of the strains.

281 In summary, this study indicates that the antimicrobial treatment allowed the emergence

282 of a resistant strain, which increased in frequency and resulted in the only $H$. parasuis

283 strain isolated, but the strain diversity could return to normality in less than a year after

284 the episode of Glässer's disease.

285

286 Acknowledgements

287 We thank Núria Galofré for technical support and the Diagnostic Service of the

288 Veterinary School at University Autònoma of Barcelona for providing us with isolate

289 228/04. This work was funded by grant AGL2004-07349 from the Ministerio de 
290 Educación y Ciencia of Spain. Fellowship support for A. O. from CReSA is also 291 acknowledged.

292

293 References

294 Altschul, S.F., Madden, T.L., Schaffer, A.A., Zhang, J., Zhang, Z., Miller, W., Lipman, 295 D.J., 1997. Gapped BLAST and PSI-BLAST: a new generation of protein 296 database search programs. Nucleic. Acids. Res. 25, 3389-3402.

297 Amano, H., Shibata, M., Kajio, N., Morozumi, T., 1994. Pathologic observations of pigs 298 intranasally inoculated with serovar 1, 4 and 5 of Haemophilus parasuis using immunoperoxidase method. J. Vet. Med. Sci. 56, 639-644.

300 Amano, H., Shibata, M., Kajio, N., Morozumi, T., 1996. Pathogenicity of Haemophilus

301 parasuis serovars 4 and 5 in contact-exposed pigs. J. Vet. Med. Sci. 58, 559302561.

303 del Rio, M.L., Gutierrez, C.B., Rodriguez Ferri, E.F., 2003. Value of indirect 304 hemagglutination and coagglutination tests for serotyping Haemophilus 305 parasuis. J. Clin. Microbiol. 41, 880-882.

306 Harris, D.L., Ross, R.F., Switzer, W.P., 1969. Incidence of certain microorganisms in 307 nasal cavities of swine in Iowa. Am. J. Vet. Res. 30, 1621-1624.

308 Janda, J.M., Abbott, S.L., 2002. Bacterial identification for publication: when is enough 309 enough? J. Clin. Microbiol. 40, 1887-1891.

310 Jolley, K.A., Feil, E.J., Chan, M.S., Maiden, M.C., 2001. Sequence type analysis and 311 recombinational tests (START). Bioinformatics 17, 1230-1231.

312 Kielstein, P., Rapp-Gabrielson, V.J., 1992. Designation of 15 serovars of Haemophilus 313 parasuis on the basis of immunodiffusion using heat-stable antigen extracts. $\mathrm{J}$.

314 Clin. Microbiol. 30, 862-865. 
315 Larsen, J.L., Bille, N., Nielsen, N.C., 1973. Occurrence and possible role of Moraxella

316 species in pigs. Acta Pathol. Microbiol. Scand. [B] Microbiol. Immunol. 81, $317 \quad 181-186$.

318 Miniats, O.P., Smart, N.L., Metzger, K., 1986. Glässer's disease in southwestern 319 ontario. I. A retrospective study. In: International Congress of the Pig $320 \quad$ Veterinarian Society, p. 279.

321 Møller, K., Kilian, M., 1990. V factor-dependent members of the family 322 Pasteurellaceae in the porcine upper respiratory tract. J. Clin. Microbiol. 28, $323 \quad 2711-2716$.

324 Nicolet, J., 1968. On haemophilosis of swine. I. Identification of a frequent agent: 325 Haemophilus parahaemolyticus. Pathol. Microbiol. 31, 215-225.

326 Nielsen, R., 1993. Pathogenicity and immunity studies of Haemophilus parasuis 327 serotypes. Acta Vet. Scand. 34, 193-198.

328 Nielsen, R., Danielsen, V., 1975. An outbreak of Glässer's disease: Studies on etiology, 329 serology and the effect of vaccination. Nord. Vet. Med. 27, 20-25.

330 Oliveira, S., Blackall, P.J., Pijoan, C., 2003. Characterization of the diversity of 331 Haemophilus parasuis field isolates by use of serotyping and genotyping. Am. J. 332 Vet. Res. 64, 435-442.

333 Oliveira, S., Galina, L., Pijoan, C., 2001. Development of a PCR test to diagnose 334 Haemophilus parasuis infections. J. Vet. Diagn. Inv est. 13, 495-501.

335 Oliveira, S., Pijoan, C., 2004. Haemophilus parasuis: new trends on diagnosis, 336 epidemiology and con trol. Vet. Microbiol. 99, 1-12.

337 Olvera, A., Calsamiglia, M., Aragon, V., 2006a. Genotypic Diversity of Haemophilus 338 parasuis Field Strains. Appl. Environ. Microbiol. 72, 3984-3992. 
339 Olvera, A., Cerdà-Cuéllar, M., Aragon, V., 2006b. Study of the population structure of

$340 \quad$ Haemophilus parasuis by multilocus sequence typing. Microbiol. 152, 3683-

$341 \quad 3690$.

342 Olvera, A., Segalés, J., Aragon, V., 2006c. Update on the diagnosis of Haemophilus parasuis infection in pigs and novel genotyping methods. Vet. J. doi:10.1016/j.tvj1.2006.10.017

Ooyen, A.v., 2001. New approches for the generation and analysis of microbial fingerprints. Elsevier, Amsterdam, 31-45 pp.

Rafiee, M., Bara, M., Stephens, C.P., Blackall, P.J., 2000. Application of ERIC-PCR for the comparison of isolates of Haemophilus parasuis. Aust. Vet. J. 78, 846-849.

Rapp-Gabrielson, V.J., Gabrielson, D.A., Schamber, G.J., 1992. Comparative virulence of Haemophilus parasuis serovars 1 to 7 in guinea pigs. Am. J. Vet. Res. 53, 987-994.

Rapp-Gabrielson, V.J., Oliveira, S.R., Pijoan, C., 2006. Haemophilus parasuis, In Straw, B.E., Zimmerman, J.J., D'Allaire, S., Taylor, D. J. (Eds.), Diseases of Swine, Blackwell publishing, Iowa, pp.681-690.

Rosner, H., Kielstein, P., Muller, W., Rohrmann, B., 1991. Relationship between serotype, virulence and SDS-PAGE protein patterns of Haemophilus parasuis. Dtsch. Tierarztl. Wochenschr. 98, 327-330.

Ruiz, A., Oliveira, S., Torremorell, M., Pijoan, C., 2001. Outer membrane proteins and DNA profiles in strains of Haemophilus parasuis recovered from systemic and respiratory sites. J. Clin. Microbiol. 39, 1757-1762.

361 Smart, N.L., Hurnik, D., Macinnes, J.I., 1993. An investigation of enzootic Glässer's 362 disease in a specific pathogen free grower-finisher facility using restriction 363 endonclease analysis. Can. Vet. J. 34, 487-490. 
364 Smart, N.L., Miniats, O.P., MacInnes, J.I., 1988. Analysis of Haemophilus parasuis isolates from southern Ontario swine by restriction endonuclease fingerprinting.

Smart, N.L., Miniats, O.P., Rosendal, S., Friendship, R.M., 1989. Glasser's disease and 368 prevalence of subclinical infection with Haemophilus parasuis in swine in southern Ontario. Can. Vet. J. 30, 1989.

370

371

\section{$372 \quad$ Figure legends}

373 Figure 1. Genotyping and serotyping of $H$. parasuis isolates. ERIC-PCR fingerprints 374 were analyzed by UPGMA and MLST allelic profiles by neighbour-joining. MLST 375 allelic profiles correspond to the alleles of $r p o B, 6 p g d, m d h$, infB, frdB, g3pd and atpD. 376 Strain 228/04 was isolated form lung lesions during a Glässer's disease outbreak, CD 377 isolates were obtained from nasal swabs after 1 month of amoxicillin treatment and CN 378 isolates were obtained from nasal swabs 1 year later. Isolates corresponding to a single 379 strain are included in the same box. ${ }^{*} \mathrm{ND}$, not determined.

381 Figure 2. Neighbour-joining dendogram constructed using the allelic profiles of loci 382 rpoB, $6 p g d, m d h, \inf B, f r d B, g 3 p d$ and $a t p D$ of $205 H$. parasuis isolates. Isolates from

383 the case farm are indicated in the dendogram. Additionally, non-virulent reference 384 strains D74, SW114, C5 and virulent reference strains Nagasaki, H367, 84-22113, 8438515995 are also indicated. 
Table 1. Antibiotic susceptibility of the different isolates studied.

\begin{tabular}{|c|c|c|c|c|c|c|c|c|}
\hline \multirow[b]{2}{*}{ Isolate } & \multirow[b]{2}{*}{ Bacteria } & \multicolumn{6}{|c|}{ Antimicrobials ${ }^{1,2}$} & \multirow[b]{2}{*}{ Isolation date } \\
\hline & & AMOXY & $\mathbf{T R}+\mathbf{S U}$ & ENROF & DOXYC & CFTIO & TYLOS & \\
\hline $228 / 04$ & H. parasuis & S (52) & S (39) & S (41) & S (39) & S (57) & S (33) & $10 / 2004$ \\
\hline CD7-3 & H. parasuis & $\mathrm{R}(0)$ & S (34) & S (34) & $\mathrm{S}(32)$ & S (38) & S (28) & $11 / 2004$ \\
\hline CD9-1 & H. parasuis & $\mathrm{R}(0)$ & S (28) & S (34) & $\mathrm{S}(32)$ & S (38) & S (34) & $11 / 2004$ \\
\hline CD10-4 & H. parasuis & $\mathrm{R}(0)$ & S (30) & S (36) & S (34) & $S(42)$ & S (30) & $11 / 2004$ \\
\hline CD11-4 & H. parasuis & $\mathrm{R}(0)$ & S (34) & S (32) & $\mathrm{S}(30)$ & S (38) & $S(26)$ & $11 / 2004$ \\
\hline CD8-1 & H.parasuis & $\mathrm{R}(0)$ & $\mathrm{S}(28)$ & S (36) & S (34) & S (42) & $\mathrm{S}(32)$ & $11 / 2004$ \\
\hline CN2-1 & H. parasuis & $\mathrm{R}(12)$ & S (32) & S (34) & S (32) & S (38) & S (26) & $10 / 2005$ \\
\hline CN6-1 & H. parasuis & I (18) & S (34) & S (34) & $S(32)$ & S (38) & $S(26)$ & $10 / 2005$ \\
\hline CN7-2 & H. parasuis & I (17) & S (34) & S (36) & $\mathrm{S}(30)$ & $S(42)$ & S (28) & $10 / 2005$ \\
\hline CN8-1 & H. parasuis & I ( 17$)$ & S (32) & S (36) & $\mathrm{S}(32)$ & S (40) & $\mathrm{S}(28)$ & $10 / 2005$ \\
\hline CN9-2 & H. parasuis & S (34) & S (30) & $S(42)$ & S (34) & $S(36)$ & $S(26)$ & $10 / 2005$ \\
\hline CN10-1 & H. parasuis & $\mathrm{R}(0)$ & S (28) & S (34) & $\mathrm{S}(32)$ & S (38) & $S(26)$ & $10 / 2005$ \\
\hline CD12CA4 & Moraxella spp & R (13) & $\mathrm{R}(15)$ & I (18) & I (24) & S (24) & $\mathrm{S}(25)$ & $11 / 2004$ \\
\hline
\end{tabular}

' AMOXY: amoxicillin $(30 \mu \mathrm{g})$; DOXYC: doxycycline (80 $\mu \mathrm{g})$; ENROF: enrofloxacin $(10 \mu \mathrm{g})$;

TR+SU: trimethoprim-su phomethoxazole $(5.2+240 \mu \mathrm{g})$; CFTIO: ceftifour $(30 \mu \mathrm{g})$; TYLOS: tylosin $(150 \mu \mathrm{g})$.

${ }^{2}$ Inhibition diameter in $\mathrm{mm}$ is indicated in parenthesis. 

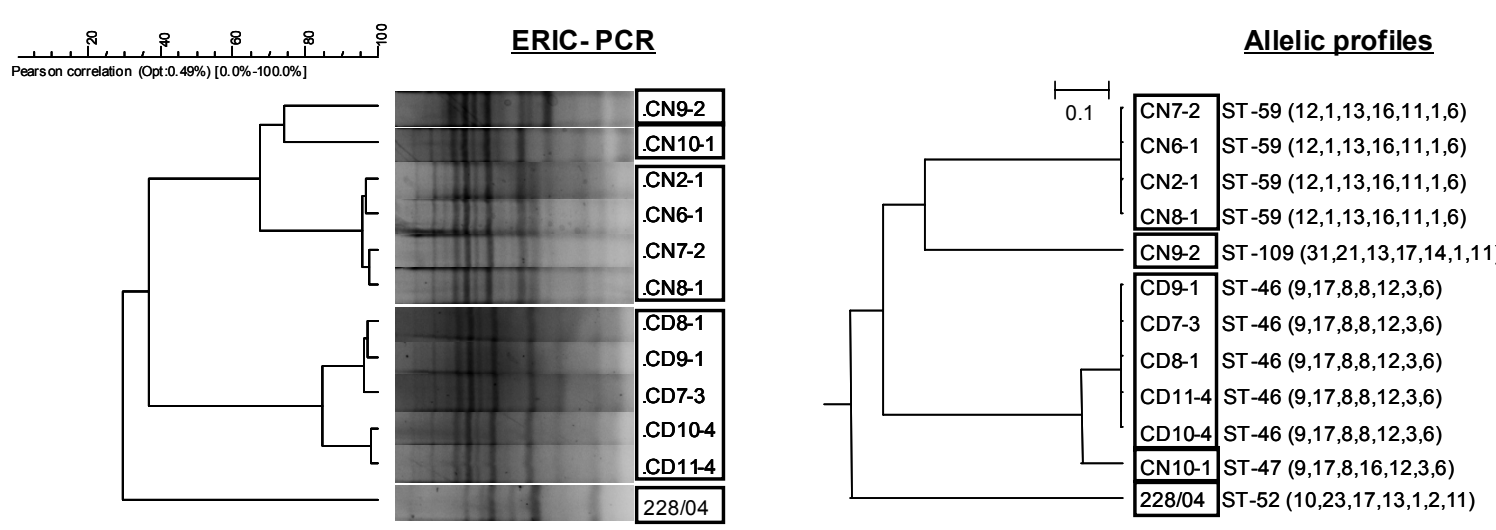

Serotype

ND*

ND

ND

9

15

15

14

ND

ND

10

15
5

Page 18 of 19 


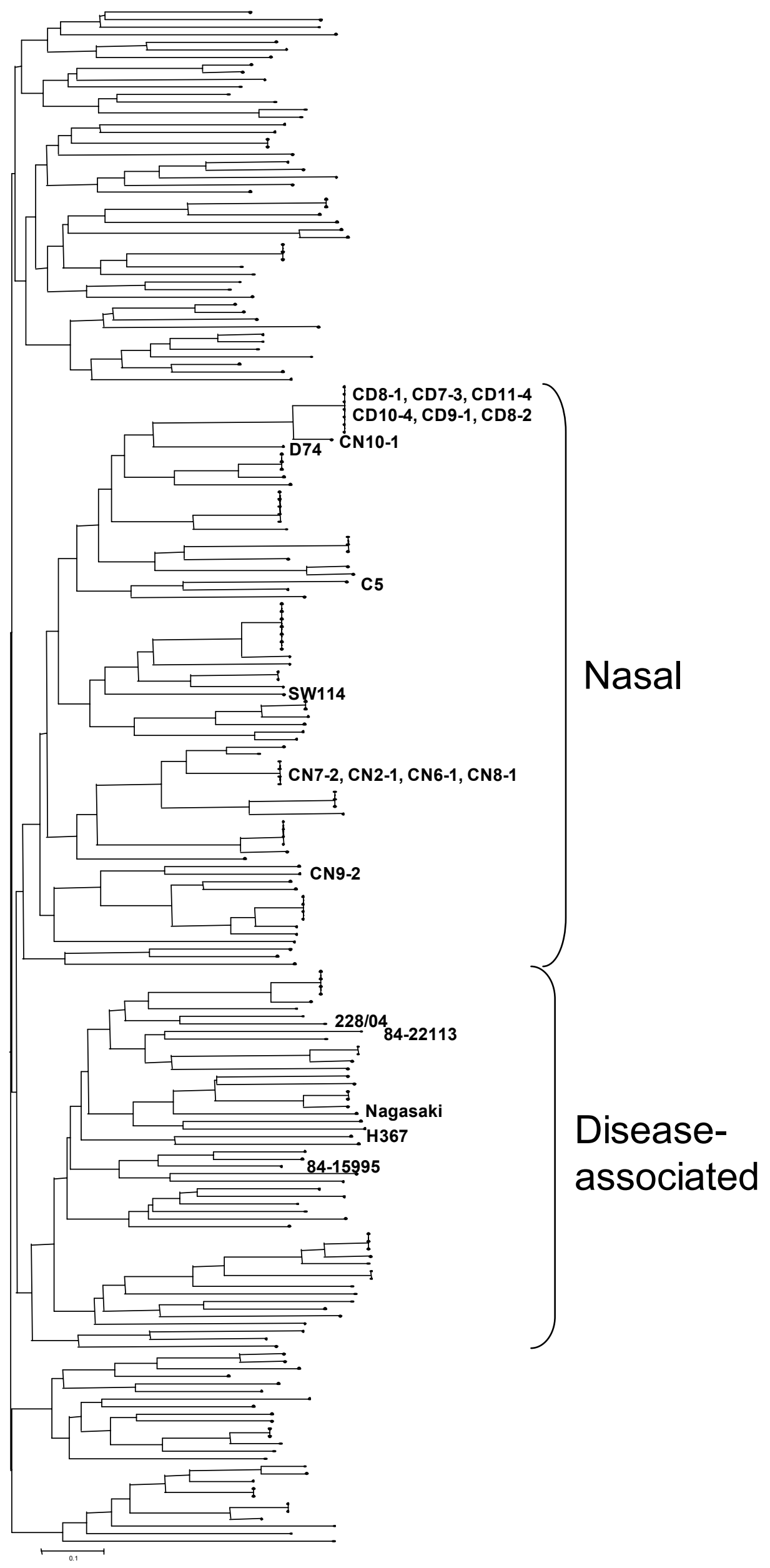

